\title{
Lunar Radar Sounder (LRS) experiment on-board the SELENE spacecraft
}

\author{
Takayuki Ono and Hiroshi Oya \\ Department of Astronomy and Geophysics, Tohoku University, Sendai 980-8578, Japan \\ (Received March 23, 2000; Revised August 11, 2000; Accepted September 1, 2000)
}

\begin{abstract}
The Lunar Radar Sounder (LRS) experiment on-board the SELENE (SELenological and ENngineering Explorer) spacecraft has been planned for observation of the subsurface structure of the Moon, using HF radar operating in the frequency range around $5 \mathrm{MHz}$. The fundamental technique of the instrumentation of LRS is based on the plasma waves and sounder experiments which have been established through the observations of the earth's magnetosphere, plasmasphere and ionosphere by using EXOS-B (Jikiken), EXOS-C (Ohzora) and EXOS-D (Akebono) satellites; and the plasma sounder for observations of the Martian ionosphere as well as surface land shape are installed on the Planet-B (Nozomi) spacecraft which will arrive at Mars in 2003. For the exploration of lunar subsurface structures applying the developed sounder technique, discrimination of weak subsurface echo signals from intense surface echoes is important; to solve this problem, a frequency modulation technique applied to the sounder RF pulse has been introduced to improve the resolution of range measurements. By using digital signal processing techniques for the generation of the sounder RF waveform and on-board data analyses, it becomes possible to improve the $\mathrm{S} / \mathrm{N}$ ratio and resolution for the subsurface sounding of the Moon. The instrumental and theoretical studies for developing the LRS system for subsurface sounding of the Moon have shown that the LRS observations on-board the SELENE spacecraft will give detailed information about the subsurface structures within a depth of $5 \mathrm{~km}$ from the lunar surface, with a range resolution of less than $75 \mathrm{~m}$ for a region with a horizontal scale of several tens of km. This capability is evaluated to be sufficient for study of the thermal history of the lunar surface region relating to a time scale of several tens of millions of years.
\end{abstract}

\section{Introduction}

The SELENE mission has been planned to study the origin and evolution processes of the Moon by using the remote sensing techniques from a polar orbit at an altitude of 100 $\mathrm{km}$. To understand the origin and evolution of the Moon, knowledge of topographical features of the subsurface to a depth of several km below the surface, with a horizontal scale of several tens of $\mathrm{km}$ is necessary; that is, the topographical and geological features of the lunar surface are strictly related to the history of lunar geology which can be read from the subsurface features (Yamaji et al., 1998). Based on results of the data analysis of the Apollo radar experiments and other studies on lunar rocks and soils, it has been ascertained that the surface layer of the Moon consists of material with a median loss tangent of 0.008 (Olhoeft and Strangway, 1975; Strangway and Olhoeft, 1977). Therefore, electromagnetic waves in the HF range can penetrate into the lunar subsurface to a depth of several $\mathrm{km}$, if the loss tangent remains constant. When geological interfaces with sharp boundaries of permittivity are encountered in the propagation path, a partial reflection of the electromagnetic waves will take place, generating subsurface echoes. Based on this concept, a radar sounder experiment ALSE (Apollo Lunar Sounder Experiment) was tested during the Apollo 17 mission for several

* Now at Fukui University of Technology.

Copy right (c) The Society of Geomagnetism and Earth, Planetary and Space Sciences (SGEPSS); The Seismological Society of Japan; The Volcanological Society of Japan; The Geodetic Society of Japan; The Japanese Society for Planetary Sciences. orbits (Phillips et al., 1973). The ALSE experiment verified that radar sounding by using HF range electromagnetic waves can be used to explore the subsurface structure of the Moon, and the results give important information for investigation of lunar tectonics within a depth of several km (Porcello et al., 1974; Sharpton and Head, 1982; Cooper et al., 1994). Since Apollo missions, there have been no additional experiments using the radar sounder technique for lunar subsurface exploration. Therefore, the Lunar Radar Sounder (LRS) is being planned to conduct a full mapping of lunar subsurface.

In the time period of the Apollo missions, there were technical limits in the space borne radar sounder techniques, especially for generating an accurate waveform of frequency modulated sounder signal, due to usage of a voltage controlled oscillator (VCO), and due to the low $\mathrm{S} / \mathrm{N}$ ratio of the data caused by the analog (optical) data recording method (Phillips et al., 1973; Peeples et al., 1978). The usage of a VCO has limit of control of a phase of RF waveform, although the specified linearity of the VCO used in the ALSE instrument had the accuracy of about $0.1 \%$. It is needed to realize a direct waveform generator, which is able to generate the same waveform for each transmission of RF pulses. An optical recording on photo-film had limit of the dynamic range of about $25 \mathrm{~dB}$. Then, it is also needed to realize a digitalized method for the data analysis and recording. We can improve the instrumentation by adopting new technology developed by the plasma sounder experiments which have been established through the plasma sounding of earth's magnetosphere, plasmasphere and ionosphere by using satel- 
lites EXOS-B (Jikiken) (Oya et al., 1981; Oya and Ono, 1981, 1987), EXOS-C (Ohzora) (Oya et al., 1985; Obara and Oya, 1985) and EXOS-D (Akebono) (Oya et al., 1990). The concept of the plasma sounder is originated in the topside sounder satellites of Alouette and ISIS (Franklin and MacLean, 1969; Jackson and Warren, 1969), namely the plasma sounder has capability of transmission of high power RF pulses into plasma through long deployed antennas. Detection of plasma sounder echo signals makes it possible to measure the plasma density profiles along the propagation path of sounder RF pulses. In addition to the sounder operation, the plasma sounder system has extended function observing detailed feature of natural plasma waves and planetary radio waves. The plasma sounder system has further been developed for the sounding of Martian ionosphere and land shape in the sounder experiment on-board the Planet-B (Nozomi) spacecraft which will start observation after the insertion into the Martian orbit in 2003 (Ono et al., 1998; Oya and Ono, 1998). The present LRS system on-board the SELENE has extended capability of sounder operation detecting subsurface echoes, in addition, it will also be able to observe the spectrum and polarization of radio wave emissions from the Earth, Jupiter, sun and other planets.

The purpose of this paper is to describe a newly developed method to achieve a high range resolution, and to describe the instrumentation of the LRS experiment on-board the SELENE spacecraft. The importance of the controlling the pulse shape is discussed, and plasma effect on the subsurface sounding signal is also evaluated in this paper.

\section{Observation Method in the LRS System}

\subsection{Estimation of intensities of surface and subsurface} echoes

The intensity of subsurface echoes largely depends on the attenuation of the transmitted RF pulses during their propagation through the lunar subsurface. The feasibility of exploration of lunar subsurface to a depth of several $\mathrm{km}$ depends on this attenuation. Most of the Earth's surface region is covered by wet soils and rocks, which prevent the penetration of electromagnetic waves at depth due to propagation losses. However, the lunar surface is covered by dry regolith material of very low conductivity. Therefore, electromagnetic waves can penetrate into a deep subsurface region and reflect back as an echo from any sharp boundary at which the electric permittivity displays a sharp discontinuity. Subsurface discontinuities may result from various geological processes; for example, the lunar mare region is thought to be due to lava eruptions that covered older surfaces, and there can be a sharp boundary between the older terrain and the mare material at a depth of several $\mathrm{km}$ below the mare surface.

Based on Maxwell's equation, applied to lunar rocks and soils which have the permeability of free space $\left(\mu_{0}\right)$, with the electric conductivity of $\sigma$ and permittivity $\epsilon$; namely,

$$
\begin{aligned}
\operatorname{rot} \vec{E} & =-\frac{\partial \vec{B}}{\partial t}=-\mu_{0} \frac{\partial \vec{H}}{\partial t} \\
\operatorname{rot} \vec{H} & =\frac{\partial \vec{D}}{\partial t}+\vec{J}=\epsilon \frac{\partial \vec{E}}{\partial t}+\sigma \vec{E}
\end{aligned}
$$

where the conductivity of a material is transformed to the

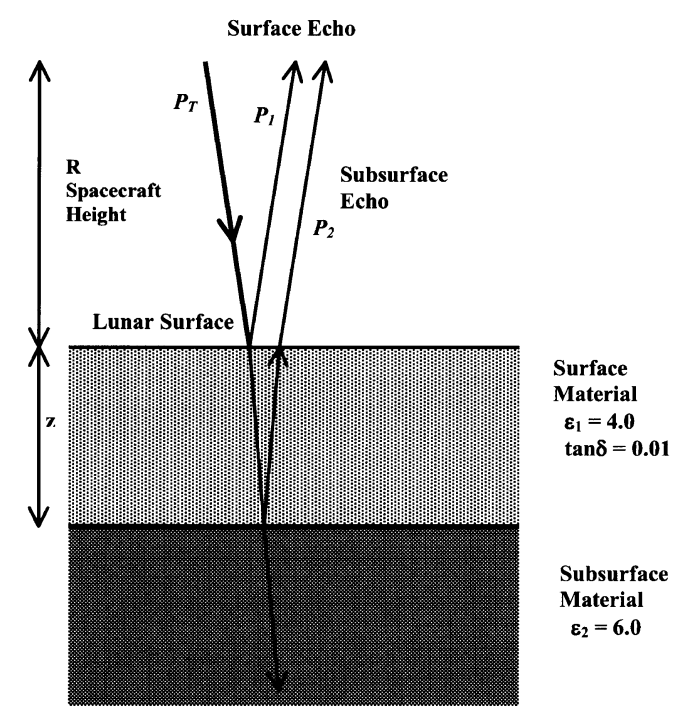

Fig. 1. A model for the transmission and penetration of electromagnetic waves of LRS sounder pulse signals at the lunar surface. We assume, in this figure, the surface material with the permittivity of 4.0 and the loss tangent value of 0.01 . This model gives subsurface material appearing at the depth of $z$, which has the permittivity of 6.0. The reflected waves at the surface give the surface echoes and the waves reflected at the subsurface boundary passing through the surface layer give the subsurface echoes.

imaginary part of the relative permittivity noted as

$$
\epsilon^{*}=\epsilon^{\prime}\left(1+\frac{i \sigma}{\epsilon \omega}\right),
$$

where $\epsilon^{*}, \epsilon^{\prime}$, and $\omega$ are a complex relative permittivity, a relative dielectric constant normalized by the permittivity of free space $\left(\epsilon_{0}\right)$, and a measured angular frequency, respectively. The loss tangent value, usually denoted as $\tan \delta$, is defined as

$$
\tan \delta=\frac{\sigma}{\epsilon \omega} .
$$

Due to a complex frequency dependence of the electric conductivity, the $\tan \delta$ value is usually measured at various frequencies, as has been reported for samples of lunar rocks and soils (Olhoeft and Strangway, 1975; Strangway and Olhoeft, 1977). They reported that the relative permittivity was measured in a range from 1.60 to 11.0 depending mainly on the bulk density of the material. The $\tan \delta$ values they measured were in a range from 0.0003 to 0.03 depending on the measured frequency ranging from $10^{2}$ to $10^{9} \mathrm{~Hz}$. Even the driest rocks have slight attenuation effects that are inversely proportional to the squares of the wavelengths of incoming radio waves; therefore, using electromagnetic waves in a low frequency range will have a significant advantage for subsurface exploration.

Intensity of the surface and subsurface echoes can be estimated by using a simplified radar equation (Phillips et al., 1973) applying Snell's law for a vertical incident electromagnetic wave on a flat surface as has been shown in Fig. 1. When we assume altitude of the satellite as $R$ and the thickness of the top layer as $z$, the intensity of the surface echo can be estimated as

$$
P_{1}=\frac{P_{T} G^{2} \lambda^{2}}{4(4 \pi R)^{2}} r_{0,1},
$$




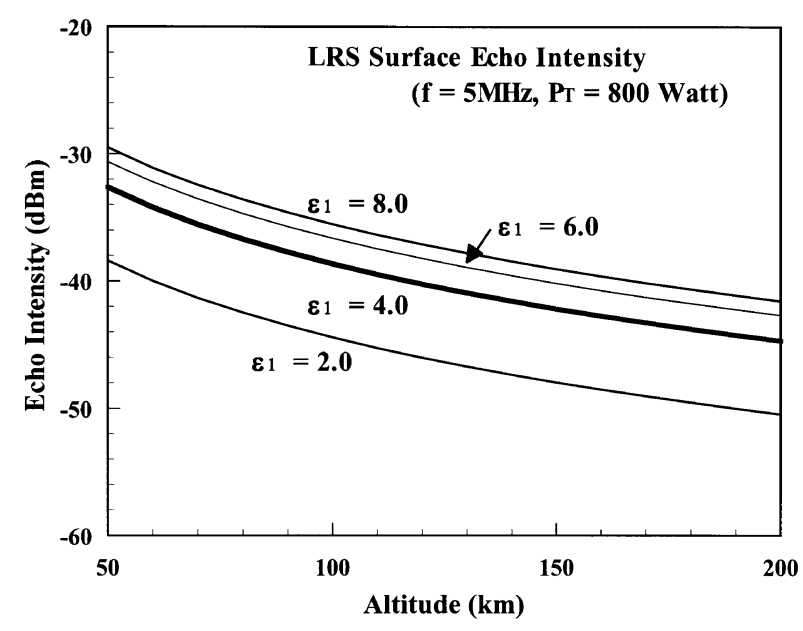

Fig. 2. Calculated intensity variations of surface echoes according to the lunar surface model shown in Fig. 1, depending on the altitude of the observation point as well as the permittivity of the surface material $\left(\epsilon_{1}\right)$ assuming the radiation power of the LRS transmission as 800 Watts at the frequency of $5 \mathrm{MHz}$.

and the intensity of the subsurface echoes is approximated as

$$
\begin{aligned}
P_{2}= & \frac{P_{T} G^{2} \lambda^{2}}{4\left\{4 \pi\left(R+R_{D}\right)\right\}^{2}}\left\{1-r_{0,1}\right\}^{2} \\
& \cdot \exp \left(-\tau_{D} \omega \tan \delta\right) r_{1,2},
\end{aligned}
$$

(Phillips et al., 1973) where $P_{T}, G, \lambda, \omega$, and $\tan \delta$ are the transmission power of sounder RF pulses, the observation antenna gain, the wavelength, the observation angular frequency, and the loss tangent value of materials of the top layer, respectively. Moreover,

$$
\begin{aligned}
R_{D} & =z \sqrt{\epsilon_{1}}, \\
\tau_{D} & =\frac{2 R_{D}}{c}, \\
r_{0,1} & =\left\{\frac{1-\sqrt{\epsilon_{1}}}{1+\sqrt{\epsilon_{1}}}\right\}^{2},
\end{aligned}
$$

and

$$
r_{1,2}=\left\{\frac{\sqrt{\epsilon_{1}}-\sqrt{\epsilon_{2}}}{\sqrt{\epsilon_{1}}+\sqrt{\epsilon_{2}}}\right\}^{2},
$$

where $\epsilon_{1}$ and $\epsilon_{2}$ are real part of relative permittivities of the top and second layers, respectively. Thus, the intensity of a surface echo is mainly dependent on the propagation range $R$, the observation wavelength $\lambda=2 \pi c / \omega$, antenna gain $G$ ( $G \approx 1.64$ for a matched dipole antenna condition) and transmission power of the sounder RF pulses $P_{T}$. The relative intensity of subsurface echoes compared to the surface echoes is given as;

$$
\frac{P_{2}}{P_{1}}=\frac{R^{2}}{\left(R+R_{D}\right)^{2}} \cdot \frac{r_{1,2}\left\{1-r_{0,1}\right\}^{2}}{r_{0,1}} \cdot \exp \left(-\tau_{D} \omega \tan \delta\right),
$$

namely, the intensity of the subsurface echoes is mainly dependent on the depth of the subsurface boundary, the observation frequency, and the loss tangent of the material of the top layer, which appears in the exponential term of the Eq.

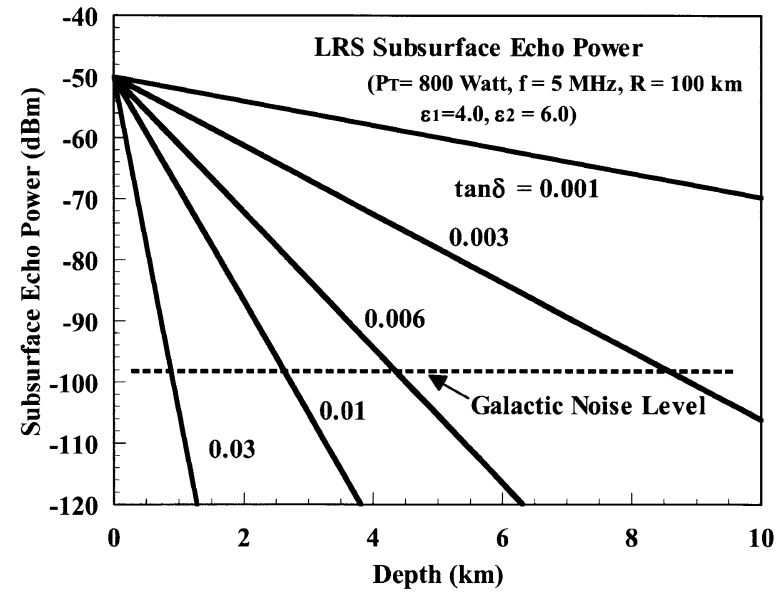

Fig. 3. Calculated intensity variations of subsurface echoes according to the lunar surface model shown in Fig. 1, depending on the depth of the subsurface layer as well as the loss tangent value of the surface material $(\tan \delta)$ ranging from 0.001 to 0.03 . The observation point of the LRS system is assumed to be at the height of $100 \mathrm{~km}$ and surface and subsurface permittivities are assumed as 4.0 and 6.0, respectively. Other conditions are assumed to be the same as the condition given in Fig. 2.

(6). Therefore, sounder echoes from deep subsurface region become observable when we select sufficiently low observation frequencies. Using electromagnetic waves in the $\mathrm{HF}$ range has a significant advantage for subsurface exploration. When we assume relative permittivities of the surface materials of the Moon in a range from 2 to 8 , and loss tangent values in a range from 0.001 to 0.03 for the HF frequency band (Olhoeft and Strangway, 1975; Strangway and Olhoeft, 1977), the calculated intensity of surface reflection echoes is shown in Fig. 2, where transmitted RF power is assumed to be 800 watts with an observation frequency of $5 \mathrm{MHz}$ measured from altitude range of 50 to $200 \mathrm{~km}$. In Fig. 3, a signal intensity variation of subsurface echo is shown against the depth of the subsurface boundary depending on the loss tangent $(\tan \delta)$ value, where the observation point is set at altitude of $100 \mathrm{~km}$, and relative permittivities of the top and the second layers are assumed 4.0 and 6.0, respectively. As shown in Fig. 3, the subsurface echo intensity varies depending on the loss tangent values of the materials of the top layer, and as a function of the depth to the subsurface layer. The level of galactic noise determines a theoretical limit of the observable echo intensity. Within a frequency range of $5 \mathrm{MHz}$, the galactic noise temperature is measured as about $2.0 \times 10^{6} \mathrm{~K}$ (Harz, 1969), that is equivalent to the intensity of $-98.6 \mathrm{dBm}$ for the present sounder receiver with the frequency bandwidth of $5 \mathrm{kHz}$. Comparing the subsurface echo intensity with the galactic noise signal, as shown in Fig. 3, the limit exists in a range from 3 to $9 \mathrm{~km}$ depending on the $\tan \delta$ value of $0.003-0.01$; therefore, the planned LRS system has a maximum observation range of about $5 \mathrm{~km}$ below the lunar surface level.

\subsection{Frequency modulation method of RF sounder}

In a conventional plasma sounder observation system, a high power RF pulses with pulse width of about $100 \mu \mathrm{sec}$ is applied for the time of flight observation for reflecting echoes (Oya et al., 1981). During a given pulse transmission 
period, the RF signal has a CW (continuous wave) waveform which is detected at the same observation frequency by the receiver. This time-of-flight observation method becomes inadequate to discriminate the weak subsurface echo signals within a range of $5 \mathrm{~km}$ (equivalent to the $33 \mu \mathrm{sec}$ delay after the surface echoes) from signals of the intense surface reflection echoes. To solve this difficulty, an FM (frequency modulation) method is applied for controlling the frequency of transmission signals of pulses of the RF sounder. The transmission signal waveform is described as a function of the time, as

$$
T_{X}(t)= \begin{cases}0 & t<0 \\ \mathrm{a}(t) \cos \phi(t) & 0 \leq t \leq T_{0} \\ 0 & T_{0}<t\end{cases}
$$

where

$$
\phi(t)=\left(\omega_{0}+\frac{1}{2} s t\right) t .
$$

Variables $\omega_{0}, s$, and $\mathrm{a}(t)$ are the starting angular frequency $\left(2 \pi \times 4 \times 10^{6} \mathrm{rad} / \mathrm{sec}\right.$ is selected for the LRS system), the sweep rate of angular frequency $\left(2 \pi \times 10^{10} \mathrm{rad} / \mathrm{sec}^{2}\right)$, and a pulse shape function to define the envelope for transmitted RF pulses, respectively; and $T_{0}$ in Eq. (12) gives the sounder pulse width (200 $\mu \mathrm{sec}$ for the LRS system). Then, the transmission frequency of the sounder RF pulse is swept from $4 \mathrm{MHz}$ to $6 \mathrm{MHz}$ within the transmission time of $200 \mu \mathrm{sec}$. The pulse shape function a $(t)$ gives a sine-shape envelope for the transmission RF pulse; i.e.,

$$
\mathrm{a}(t)=\sin \left(\pi \times \frac{t}{T_{0}}\right) .
$$

When the echo arrives with a delay time of $\tau$, the detected waveform becomes;

$$
R_{X}(t)=\beta \mathrm{a}(t-\tau) \cos \phi(t-\tau)
$$

with reduction rate $\beta$.

Being synchronized with the frequency modulation of the transmitted RF pulse signal, a swept local frequency signal is generated with the same swept rate, defined as;

$$
L_{O}(t)=\cos \phi\left(t-\tau_{0}\right), \quad \text { for } \quad \tau_{0}<t<T_{0}+2 \tau_{0}
$$

where $\tau_{0}$ is the timing of the delay for the local signal after the start of frequency sweep of the transmitted RF pulse. The received signal given by Eq. (15) is mixed with the local signal given by Eq. (16) and converted to the waveform of the sounder receiver signal $R(t)$ after passing through a low-pass filter, that is;

$$
\begin{aligned}
R(t)= & \frac{\beta \mathrm{a}(t-\tau)}{2} \cos \left\{s \cdot\left(\tau-\tau_{0}\right) t\right. \\
& \left.+\left(\omega_{0}-\frac{s}{2}\left(\tau+\tau_{0}\right)\right)\left(\tau-\tau_{0}\right)\right\} .
\end{aligned}
$$

This result, then, shows that the signal frequency becomes $s \cdot\left(\tau-\tau_{0}\right)$ giving a conversion of delay time of echoes to the frequency of the sounder receiver output signal. When the signal from a surface echo has a delay time of $\tau_{1}$ and a subsurface echo has a delay time of $\tau_{2}$, then the separation

\section{Frequency - Time Diagram of LRS Operation}

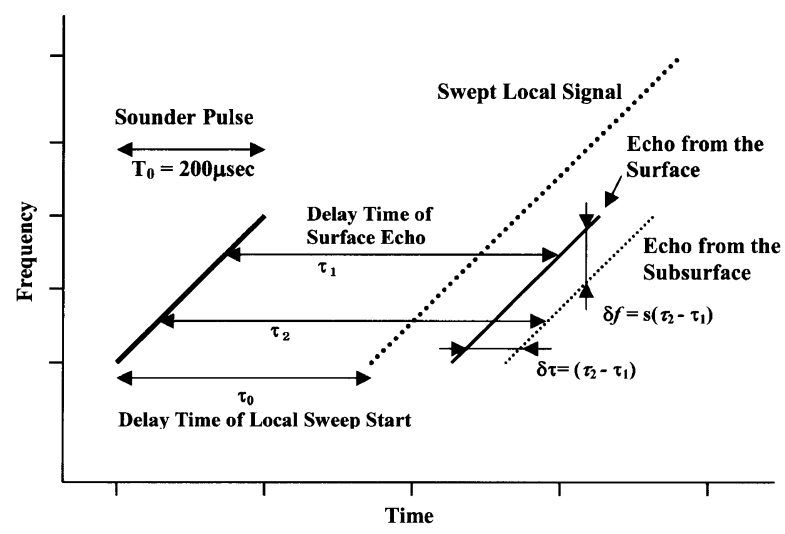

Fig. 4. A frequency versus time diagram of the LRS operation. Within the sounder pulse transmission period of $200 \mu \mathrm{sec}$, the LRS transmission frequency is swept from $4 \mathrm{MHz}$ to $6 \mathrm{MHz}$. Because SELENE spacecraft has a circular orbit with an altitude of $100 \mathrm{~km}$, a surface echo observation signal arrives with a delay time $\tau$ of about $660 \mu \mathrm{sec}$. The local signal has the same sweep rate with that of the transmission signal, with a fixed delay time of $\tau_{0}$ (it is programmable adjusting the best condition of observations) and mixed with the observation signal. As has been shown in the figure, difference of the delay time $(\delta \tau)$ will be converted to the difference in the frequency $(\delta f)$ in the LRS receiver, obtaining well time resolution as well as separation for an echo detection.

of the signal can be made by discriminating a frequency $s \cdot\left(\tau_{1}-\tau_{0}\right)$ from $s \cdot\left(\tau_{2}-\tau_{0}\right)$; that is, a frequency difference of $s$. $\left(\tau_{2}-\tau_{1}\right)$ is created (see Fig. 4). Based on Eq. (17), conversion from a frequency difference $(\delta \omega)$ to a corresponding distance $(\delta z)$ can be obtained as,

$$
\delta z=\frac{c}{2 \sqrt{\epsilon_{1}}} \frac{\delta \omega}{s},
$$

where $\epsilon_{1}$ and $c$ are the relative dielectric constant of the surface material and the speed of light, respectively. Thus, the range resolution depends on the frequency resolution of the system. In this LRS system, we employ the Fourier analysis method to analyze the frequency applying the Fast Fourier Transform (FFT) on-board the spacecraft. Based on the sampling theorem, the frequency resolution of Fourier analysis can be estimated as a function of the data-sampling period that is the same as the period of pulse transmission; i.e., the theoretical limit is $5 \mathrm{kHz}$ for the present LRS system. This frequency resolution of $5 \mathrm{kHz}$ is, then, equivalent with the range resolution of $75 \mathrm{~m}$ for a free-space medium; and the frequency resolution is equivalent to the range of $37.5 \mathrm{~m}$ for a case of propagation through a media with permittivity of 4.0 .

The selection of the shape function a $(t)$ is important to minimize the occurrence of spurious frequency spectra in the FFT analysis. Figure 5 gives examples of FFT power spectra comparing results for two shape functions. One waveform is applied a sine-shaped envelope function, and the other is a rectangular waveform (indicated as "no-shape control"). Each echo waveform consists of intense surface echo and subsurface echo of the sounder receiver output (defined by Eq. (17)) which are synthesized applying delay times of $\tau_{0}$, $\tau_{1}$, and $\tau_{2}$ as 647,667 , and $674 \mu \mathrm{sec}$, respectively, and assuming that intensity of subsurface echo is $-50 \mathrm{~dB}$ less than 

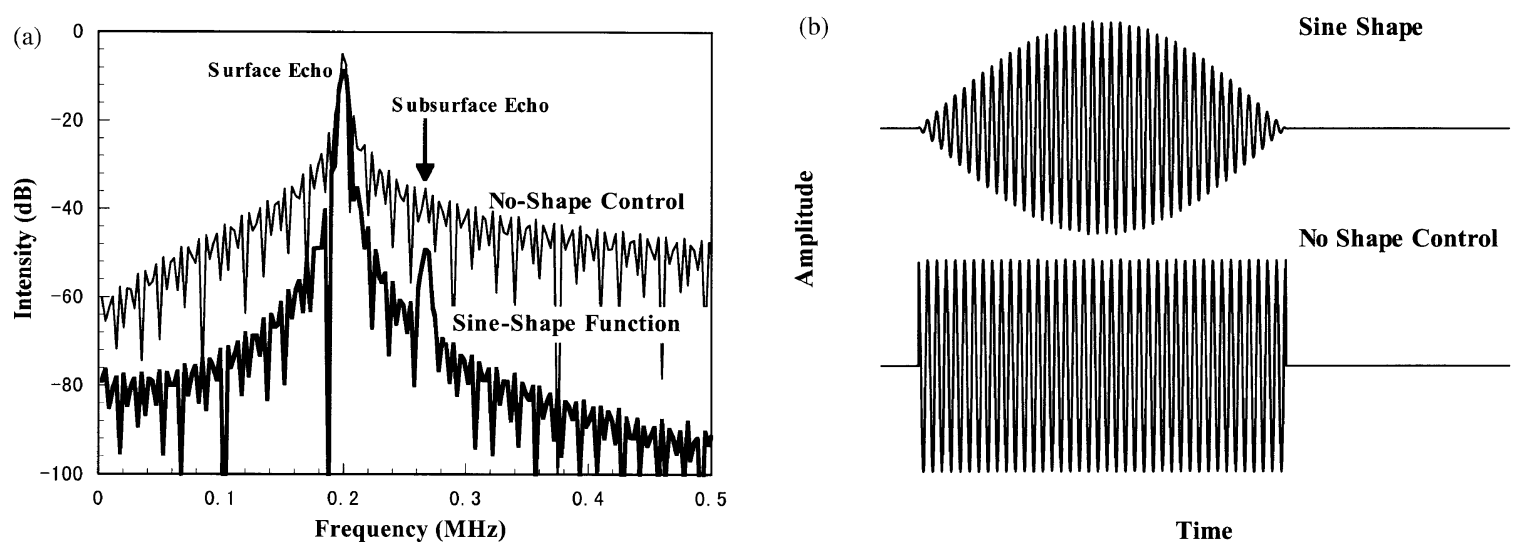

Fig. 5. A model calculation results of FFT data analyses for synthesized LRS signals which are containing subsurface echoes of weak intensities with $-50 \mathrm{~dB}$ levels of the surface echoes (plate (a)). Two curves correspond to results of different types of shape function adjusting the transmission signal envelope. Plate (b) shows waveforms of the sounder receiver defined by Eq. (17), applying the sine shape function and no shape control. In these cases, surface echoes generate receiver output signals with center frequency of $200 \mathrm{kHz}$. As has been shown in plate (a), a subsurface echo is clearly observable for the sine shape function, due to the effect of pulse envelope control.

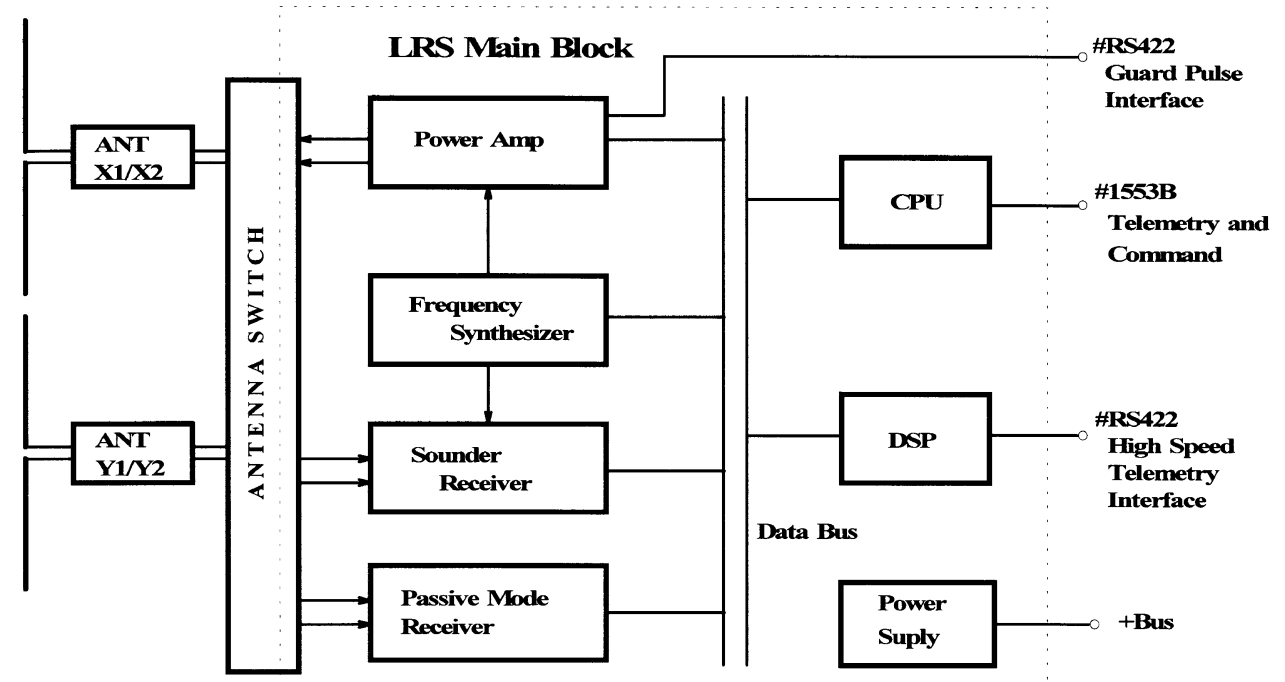

Fig. 6. A block diagram of the LRS system on-board the SELENE spacecraft. The system consists of the frequency synthesizer, the sounder power amplifier, the antenna system, the sounder receiver, the passive mode receiver, the digital signal processor, and the central processing unit for the telemetry and commands, and power supply unit. The major parts of electric circuits consist of digital signal processing devices.

the surface echo. As shown in Fig. 5, application of a sineshaped envelope function on the sounder RF pulses results in significant reduction of spurious spectra after the processing of the FFT compared with the case for the rectangular shaped pulse; by using the sine-shaped envelope, it becomes possible to find the weak echo from the subsurface boundary with a relative intensity of $-50 \mathrm{~dB}$ with respect to the echo from the surface, with the delay time of $6.7 \mu \mathrm{sec}$ that is equivalent to the depth of $500 \mathrm{~m}$ for a medium with an assumed $\epsilon_{1}=4.0$. A threshold detection level for subsurface echoes can be defined as the level of spurious frequency related to the surface echo; it is found to be about $0.23 \mathrm{MHz}$ for the case of the sine envelope shape (see Fig. 5). There is a frequency difference of $0.03 \mathrm{MHz}$ between the frequency of threshold and the peak frequency corresponding to the surface echo. The frequency gap of $0.03 \mathrm{MHz}$ corresponds to the delay time of $3.0 \mu \mathrm{sec}$. Therefore, for the present LRS system with the control of a sine-shape function for the transmitting pulse signals, a limit of detection is $3.0 \mu \mathrm{sec}$ $(225 \mathrm{~m})$ for a subsurface echo when it has a relative intensity of $-50 \mathrm{~dB}$ to the surface echoes. Thus, selecting the optimal shape function a $(t)$ for the transmission signal is important for discrimination between weak subsurface echoes and intense surface echoes. However, there is one drawback to using the sine function for $\mathrm{a}(t)$ : the power is reduced by $2 / \pi \approx 0.63(-2 \mathrm{~dB})$ (see Fig. 4). Because of power and mass limitations, the allowable output power of the RF pulse becomes 800 Watts, therefore, the energy to illuminate the lunar surface is 0.1 Joule. Among the possibilities we have examined, the shape function of sine envelope is the most 
Table 1. Major system parameters of the LRS instrument.

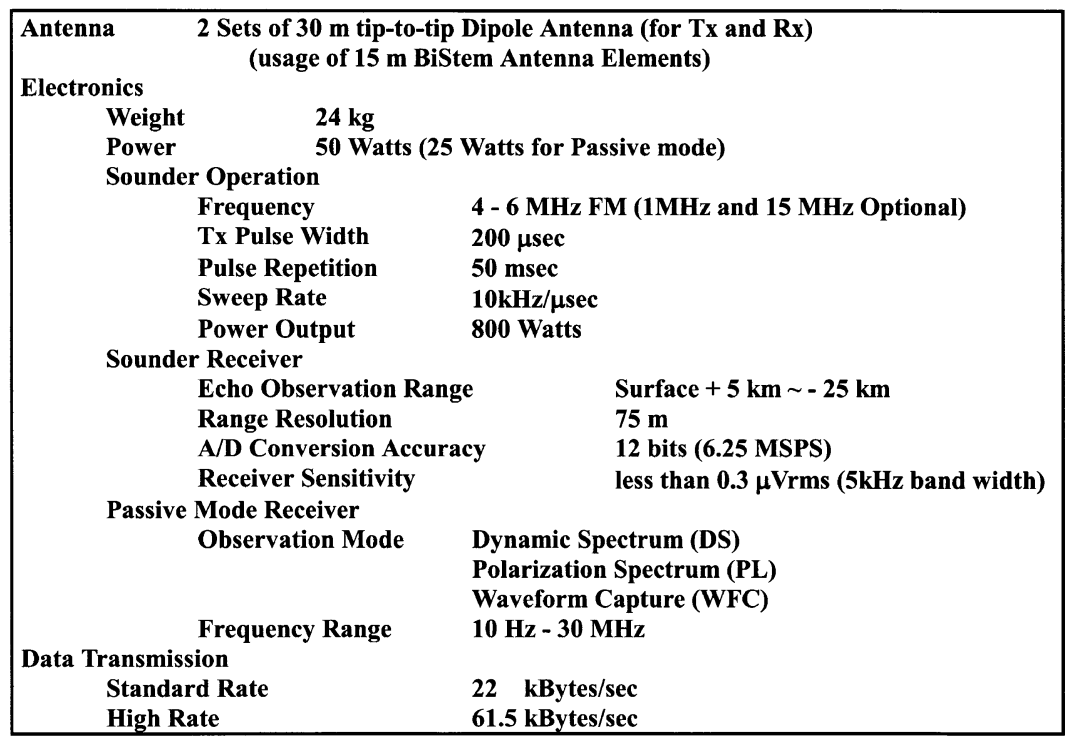

effective from the viewpoint of the frequency (range) separation performance as well as the power efficiency.

\section{Instrumentation}

The instrumentation for the Lunar Radar Sounder (LRS) experiment described in the previous section is designed on the basis of full digital signal processing, except for the transmission of RF pulse; a digital control system is employed for signal synthesizing and on-board data analysis. A block diagram of the LRS system is shown in Fig. 6. The frequencymodulated signal is fed to the power amplifier to obtain the maximum power of 800 Watts (see Table 1). The large amplitude RF pulses are fed to the dipole antenna whose tip-to-tip length is $30 \mathrm{~m}$. The echo signals from the surface and subsurface region of the Moon are detected through the other dipole antenna and fed to the sounder receiver circuit. The swept local signal is mixed with the received echo signals to convert the sounder receiver signal to the medium frequency range; the converted signals are digitized by a high-speed A/D converter with a conversion speed of $6.25 \mathrm{MHz}$ with an accuracy of 12 bits. Digital data processing including the FFT (fast Fourier transform) analysis is performed by using a DSP (digital signal processor). The LRS system is designed also for space plasma and radio physics. When the sounder transmitter is turned off, the receiver will be operated in the passive mode for observations of natural plasma waves and radio waves with a wide frequency range from 10 $\mathrm{Hz}$ to $30 \mathrm{MHz}$ aiming at the natural plasma waves surrounding the Moon and electromagnetic waves radiated from the Sun, Earth, and other planets (see Table 1).

The RF signals whose frequencies are swept from $4 \mathrm{MHz}$ to $6 \mathrm{MHz}$ within $200 \mu \mathrm{sec}$ of the transmission period (defined in Eq. (12)) is synthesized directly by using a waveform memory and a high-speed digital to analog (D/A) converter with an accuracy of 12 bits including the pulse shape control. The transmission signal is fed to the power amplifier obtaining power of 800 Watts. This high power output is realized applying a parallel operation of power amplifier circuits, which have already been developed for previous plasma sounder experiments (Oya et al., 1981). The swept frequency local signal given by Eq. (16) is also synthesized by using the same principle and subsystem. In this case, the start time $\left(\tau_{0}\right)$ of the swept frequency operation is delayed by about $600 \mu \mathrm{sec}$ and continued within a period of $400 \mu \mathrm{sec}$. The delay time $\tau_{0}$ is selectively determined by the program of the on-board computer referring the orbital condition of the SELENE spacecraft to keep $\left(\tau-\tau_{0}\right)$ being almost the same value for the nadir surface echoes. The $400 \mu \mathrm{sec}$ duration of the local signal is equivalent to an observation window of the apparent range set as $60 \mathrm{~km}$. The timing diagram for the pulse transmission and start of swept frequency of local signal and the reception of sounder echoes is illustrated in Fig. 4.

To minimize the amount of data to be transmitted to the ground, the start time of the local signal is set so as to keep a short leading time. It will be set as $20 \mu \mathrm{sec}$, for example, to keep the appearance of the surface echo at the lower frequency range in the sounder receiver signal output as shown in Fig. 5. To realize this condition, the satellite altitude is predicted by calculating orbital elements of the SELENE spacecraft, which has been designed as a circular polar orbit with altitude of $100 \mathrm{~km}$.

Two sets of dipole antennas with a tip-to-tip length of 30 $\mathrm{m}$ are used for the high power RF pulse transmission and echo signal reception. The element length of the dipole is selected to match the quarter wavelength of the electromagnetic waves at $5 \mathrm{MHz}$. Because three-axis-stabilized attitude control is designed for the SELENE satellite, a Bi-Stem antenna element is used to keep the dipole formation without spin stabilizing the spacecraft. For the case of normal mode operation, high power RF pulses are fed to one set of the dipole antenna which consists of $\mathrm{Y} 1$ and $\mathrm{Y} 2$ antenna (Y1Y2 antenna hereafter) elements while the echo signals are picked up by the other set of the dipole which consists of $\mathrm{X} 1$ and $\mathrm{X} 2$ (X1-X2 antenna hereafter) which are set in the orthogonal direction to the Y1-Y2 elements dipole antenna. 


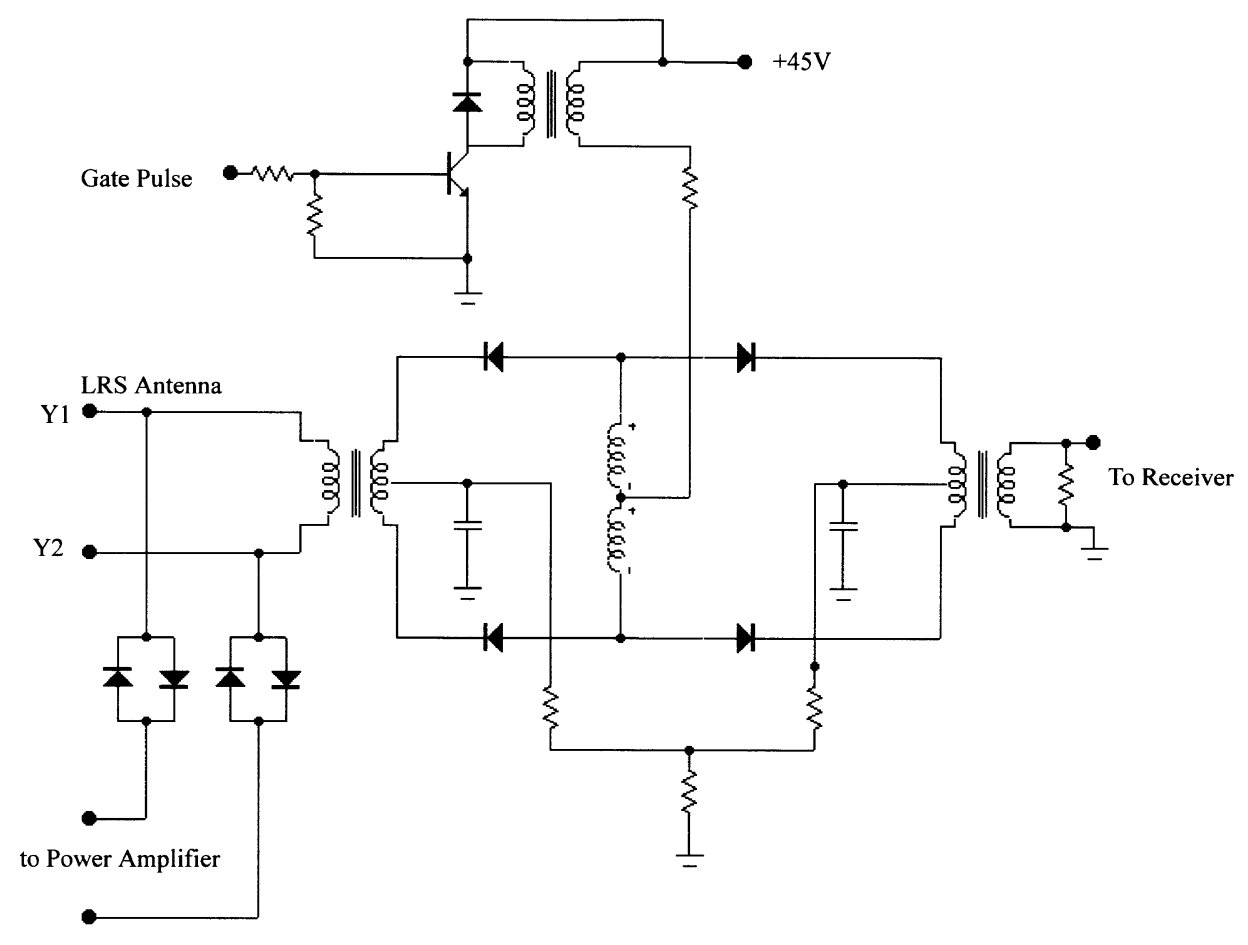

Fig. 7. The circuit diagram of the Transmitter/Receiver switch (TR switch) which is able to switch alternatively the antenna connection between the power amplifier and the receiver with a high speed and with low power losses.

For the receiving antenna, it is, however, selectable so as to switch to pick up the echo signal by the RF pulse transmission antenna (normally Y1-Y2 antenna) that makes it possible to detect the electric field component in the same direction as the transmitted electric fields. This condition, similar to a specular reflection condition, may occur for the observation above a flat plane surface of the Moon. In this case, a highspeed transmitter-receiver switch (TR switch; Franklin and MacLean, 1969) will be applied to switch off the receiver from the antenna during the RF pulse transmission period (200 $\mu \mathrm{sec})$; the circuit diagram is shown in Fig. 7.

Depending on the allowed data transmission speed of the SELENE spacecraft to the ground, the data transmission speed of the LRS system is planned to operate with two modes; namely, $22 \mathrm{kBytes} / \mathrm{sec}$ for the standard data transmission mode, and $61.5 \mathrm{kBytes} / \mathrm{sec}$ for the high-speed data transmission mode. Within the standard transmission period the sounder echo data with FFT data analysis will be transmitted to minimize the volume of data to $12 \mathrm{kBytes} / \mathrm{sec}$, on the other hand, by using the high speed data transmission mode, the full waveforms of the sounder receiver signal will be transmitted to the ground with data rate of $60 \mathrm{kBytes} / \mathrm{sec}$ for data analyses where the method of synthetic aperture analyses is applicable.

\section{Discussion}

\subsection{Plasma effect}

The propagation characteristics of the sounder RF pulses are generally subject to the dispersion effects, due to space plasma, by which the delay time $\tau$ in Eq. (15) is no longer a constant for the same range value. Because of the weakness of the magnetic field surrounding the Moon, the dispersion effect can be roughly examined under no magnetic field condition. In this case, the delay time can be described with a simple form for

$$
\tau=2 \int_{0}^{h} \frac{d s}{V_{g}}
$$

as

$$
\tau=\frac{2}{c} \int_{0}^{h}\left(1+\frac{1}{2 \omega^{2}} \frac{e^{2} N(z)}{m_{e} \epsilon_{0}}\right) d z,
$$

where $V_{g}, c, N(z)$, and $\omega$ are group velocity, the speed of light, the electron number density along the propagation path, and the angular frequency of propagating electromagnetic waves, respectively. By a simple mathematical manipulation, it follows that

$$
\tau=\frac{2 h}{c}+\frac{K}{c \omega^{2}} \int_{0}^{h} N(z) d z
$$

where

$$
K=\frac{e^{2}}{m_{e} \epsilon_{0}}=3.18 \times 10^{3} \quad \text { (in MKS unit), }
$$

and $e, m_{e}, \epsilon_{0}$ are unit of charge, electron mass, and absolute permittivity of vacuum, respectively. The integral in Eq. (21) is the total column density (noted as $N_{T}$ ) of plasma along the propagation path from the observation point $(z=0)$ to the target $(z=h)$. The delay time given in Eq. (20) can, then, be rewritten by

$$
\tau=\tau_{f}+\tau_{p},
$$

where $\tau_{f}$ and $\tau_{p}$ are the delay time due to the propagation as electromagnetic waves in vacuum and the excess time delay 
as effect of plasma in the propagating medium, respectively. These are given by

$$
\tau_{f}=\frac{2 h}{c},
$$

and

$$
\tau_{p}=\frac{K N_{T}}{c\left(\omega_{0}+s \cdot\left(t-\tau_{f}\right)\right)^{2}} .
$$

In this case, the waveform of the echo signal as given in Eq. (15) is expressed by inserting Eq. (22) to $\tau$. The modification of time $\tau_{p}$ becomes significant, if the product of $s \tau_{p}$ is not negligibly small compared with the frequency resolution of the echo signal, which is determined by the process of FFT. In the case of lunar exploration, the column density of the solar wind plasma for the distance from the surface to the SELENE spacecraft is estimated to be $N_{T}=10^{12} / \mathrm{m}^{2}$. The excess delay time is given by $\tau_{p}=1.67 \times 10^{-8} \mathrm{sec}$ for the start frequency of $4 \mathrm{MHz}$ and it is $0.74 \times 10^{-8} \mathrm{sec}$ for $6 \mathrm{MHz}$; then it is possible to ignore the effect of the solar wind plasma on the sounder observations because the resulted frequency differences are $167 \mathrm{~Hz}$ for $4 \mathrm{MHz}$ signal, and it is $74 \mathrm{~Hz}$ for $6 \mathrm{MHz}$ signal in the result of Eq. (17).

However it should be noted for the case of other planets or moon covered by dense plasma environment, the sounder observation by using the method of LRS system should be designed considering plasma effects. When we examine the case of a model ionosphere with no magnetic field whose plasma density is roughly the same order of the Earth's ionosphere, apart from the exploration of the Moon, the column density of the ionosphere often exceeds the value of $N_{T}=10^{15} / \mathrm{m}^{2}$ which gives $\tau_{p}=16.7 \mu \mathrm{sec}$ for the start frequency of $4 \mathrm{MHz}$ and gives $\tau_{p}=7.4 \mu \mathrm{sec}$ for the end time of the sounder pulse. Then, the sounder receiver signal obtained by Eq. (17) is significantly affected in their frequency linearity with a magnitude of several tens $\mathrm{kHz}$. Thus, the sounder echo signal is significantly modified in its waveform character due to the plasma dispersion effect of a dense plasma condition.

\subsection{Feasibility of detection of subsurface echoes}

The detection of the echoes reflected from the subsurface is not simply a matter of the echo's power but the subject of the $\mathrm{S} / \mathrm{N}$ ratio mainly caused by the contamination of the echoes reflected from the irregular structure of the lunar surface. To clarify the contamination effects due to scattering echoes caused by the roughness of the lunar surface, simulation studies have been started. The results will be published in a different paper in which the conclusion will be made that subsurface echoes are possible to be discriminated from intense clatter echo signals returned from the rough surface of mare regions and even from heavily-cratered terrains of highland regions by applying extended analysis methods using signal stacking of the data. Application of signal filtering methods to discriminate given ranges for remarkable reflecting echoes from surface objects is also effective to increase the $\mathrm{S} / \mathrm{N}$ ratio. Detailed descriptions for these methods are deferred to the other paper (Kobayashi et al., 2000).

\section{Conclusion}

The Lunar Radar Sounder (LRS) experiment will allow the observation of subsurface structure of the Moon for lunar ex- ploration; the basic technology for this subsurface sounder is prepared as an extension of the plasma sounder instrumentation which has been developed through satellite observations in the ionosphere, the plasmasphere and the magnetosphere, using the EXOS-B, C, and D satellites launched in the last 25 years. The subsurface is separated by a few $\mathrm{km}$ from the lunar surface, thus the discrimination of this short range is required. For this purpose, a method of frequency modulation of RF pulses is adopted. In the case of the present LRS experiment, the range resolution is $75 \mathrm{~m}$. The method has already been employed in high-resolution radar such as SAR (Synthetic Aperture Radar), and was also used for the ALSE experiment on Apollo 17. For measurements of the range of the echoes, the on-board FFT analyzer is installed in the LRS; all these systems are operated digitally. To realize the capability of high resolution for separation of the echoes for identifying weak subsurface echoes reflected from the discontinuity located close to objects, which produce relatively strong surface echoes, it is necessary to apply a pulse shaping function to the form of RF pulse. We find that a shaping form of a sine function gives suitable waveform, with low spurious spectra, for separating subsurface echoes from intense surface echoes whose frequency is closely located to that of subsurface signals, with only a small loss of the power output from the sounder transmitter.

The instrumentation of the LRS system consists of two sets of dipole antennas, the power transmitter with designed output power of 800 Watts, the frequency synthesizer for generation of waveform of the transmission signal with swept RF frequency, and a local signal also with swept frequency whose start time is controlled by adjusting the delay time range of the echo signal. The LRS system also consists of a receiver where the echo signals, whose frequency is converted to fixed center frequency signal, are mixed with the swept local signal; and the obtained fixed center frequency signal is fed to the digital signal-processing unit where the signals are analyzed by FFT within a given bandwidth. Due to the selection of observation frequency in HF range centered at $5 \mathrm{MHz}$, the tip-to-tip length of the dipole made of the $\mathrm{Bi}$-Stem elements are set at $30 \mathrm{~m}$. To increase the transmission power, two power amplifiers have been designed to be operated in parallel; these power amplifiers are based on the already-developed instrumentation of the plasma sounder experiments on-board the earth orbiting satellites and the Planet-B spacecraft for the Mars exploration with a potential capability of the stable RF power transmission of 600 Watts. Within the data transmission rate allowed for the LRS operation (22 kBytes $/ \mathrm{sec}$ or $61.5 \mathrm{kBytes} / \mathrm{sec})$, the sounder receiver generates data with the rate of $12 \mathrm{kBytes} / \mathrm{sec}$ for the case of the standard operation; the rate is increased to be $60 \mathrm{kBytes} / \mathrm{sec}$ for the high-speed operation that is selected depending on the speed of the telemetry system.

The effect of the solar wind plasma around the Moon is investigated as dispersive media for the plasma waves even in a range of high frequency signal. The dispersion effects of plasma in the propagation medium affects the frequency modulation method itself; and it becomes quite severe if the total plasma content increases as much as $5 \times 10^{13} / \mathrm{m}^{2}$. However, this effect can be negligible for the present LRS observation on the lunar orbit where expected column density of 
solar wind plasma becomes only about $10^{12} / \mathrm{m}^{2}$.

The present study verified that the planned LRS experiment on-board SELENE spacecraft is feasible for exploration of the lunar subsurface, it is shown that a subsurface echo signal from a depth of several $\mathrm{km}$ ranges is detectable for the LRS system. Although there have been results of the ALSE experiment verifying the possibility of sounding of the subsurface structure by using an HF sounder method, an extended theoretical study has been needed to verify the generalized methodology of the data analysis, and to identify the subsurface echoes from expected intense surface echoes due to a rough surface shape of the Moon including a distribution of huge numbers of craters. Computer simulations of sounder echo signals from various types of lunar surface and subsurface horizons have been done; the results of those simulations will be published in other papers.

Acknowledgments. The SELENE Mission has been conducted as a joint project between the Institute of Space and Astronautical Science (ISAS) and the National Space Development Agency of Japan (NASDA).

\section{References}

Cooper, B. L., J. L. Carter, and C. A. Sapp, New evidence for graben origin of Oceanus Procellarum from lunar sounder optical imagery, J. Geophys. Res., 99(E2), 3799-3812, 1994.

Franklin, C. A. and M. A. MacLean, The design of swept-frequency topside sounders, Proc. IEEE, Vol. 57, 897-929, 1969.

Harz, T. R., Radio noise levels within and above the ionosphere, Proc. IEEE, Vol. 57, 1042-1050, 1969.

Jackson, J. E. and E. S. Warren, Objectives, History, and Principal achievements of the topside sounder and ISIS project, Proc. IEEE, Vol. 57, 861$865,1969$.

Kobayashi, T., T. Ono, and H. Oya, Computer simulation on investigation of lunar subsurface structure by radar sounders-Studies related to the SELENE project, Earth Planets Space, 2000 (to be submitted).

Obara, T. and H. Oya, Plasma condition in the polar ionosphere observed in SPW experiments of PPS system on board the Ohzora (EXOS-C) satellite, J. Geomag. Geoelectr., 37, 285-307, 1985.

Olhoeft, G. R. and D. W. Strangway, Dielectric properties of the first 100 meters of the moon, Earth Planet. Sci. Lett., 24, 394-404, 1975.
Ono, T., H. Oya, A. Morioka, A. Kumamoto, K. Kobayashi, T. Obara, and T. Nakagawa, Plasma Waves and Sounder (PWS) experiment onboard the Planet-B Mars orbiter, Earth Planets Space, 50, 213-221, 1998.

Oya, H. and T. Ono, Stimulation of plasma waves in the magnetosphere using satellite JIKIKEN (EXOS-B), Adv. Space Res., 1, 217-220, 1981.

Oya, H. and T. Ono, Stimulation of plasma waves in the magnetosphere using satellite JIKIKEN (EXOS-B) part II: Plasma density across the plasmapause, J. Geomag. Geoelectr., 39, 591-607, 1987.

Oya, H. and T. Ono, A new altimeter for Mars land shape observations utilizing the ionospheric sounder system onboard the Planet-B spacecraft, Earth Planets Space, 50, 229-234, 1998.

Oya, H., T. Ono, and T. Kamada, Stimulation of plasma waves in the magnetosphere using satellite JIKIKEN (EXOS-B), Part-I: Observation of plasma resonances, J. Geomag. Geoelectr., 33(1), 3-25, 1981.

Oya, H., A. Morioka, K. Kobayashi, M. Iizima, T. Ono, H. Miyaoka, T. Okada, and T. Obara, Plasma wave observations and sounder experiments (PWS) on-board Akebono (EXOS-D) satellite, J. Geomag. Geoelectr., 42, 411-442, 1990.

Oya, H., A. Morioka, and T. Obara, Leaked AKR and Terrestrial hectometric radiations discovered by the plasma wave and planetary plasma sounder experiments on-board the Ohzora (EXOS-C) satellite-instrumentation and observation results of plasma wave phenomena, J. Geomag. Geoelectr., 37, 237-262, 1985.

Peeples, W. J., W. R. Sill, W. M. Thomas, S. H. Ward, R. J. Phillips, R. L. Jordan, E. A. Abbott, and T. J. Killpack, Orbital radar evidence for lunar subsurface layering in Maria Serenitaris and Crisium, J. Geophys. Res., 83(B7), 3459-3468, 1978.

Phillips, R. J., G. F. Adams, W. E. Brown, Jr., R. E. Eggleton, P. L. Jackson, R. Jordan, W. I. Linlor, W. J. Peeples, L. J. Porcello, J. Ryu, G. Schaber, W. R. Sill, T. W. Thompson, S. H. Ward, and J. S. Zelenka, Apollo lunar sounder experiment, NASA Spec. Publ., 330(22), 1-26, 1973.

Porcello, L. J., R. L. Jordan, J. S. Zelenka, G. F. Adams, R. J. Phillips, W. E. Brown, Jr., S. H. Ward, and P. L. Jackson, The Apollo lunar sounder radar system, Proc. IEEE, Vol. 62, 769-783, 1974.

Sharpton, V. L. and J. W. Head III, Stratigraphy and structural evolution of southern Mare Serenitaris: a reinterpretation based on Apollo lunar sounder experiment data, J. Geophys. Res., 87(B13), 10983-10998, 1982.

Strangway, D. W. and G. R. Olhoeft, Electrical properties of planetary surfaces, Phil. Trans. R. Soc. London, A, 285, 441-450, 1977.

Yamaji, A., S. Sasaki, Y. Yamaguchi, T. Ono, J. Haruyama, and T. Okada, Lunar tectonics and its implications for the origin and evolution of the moon, Mem. Geol. Soc. Japan, 50, 213-226, 1998.

T. Ono (e-mail: ono@stpp3.geophys.tohoku.ac.jp) and H. Oya 